\title{
Cognitive factors and adaptation to auditory-visual discordance
}

\author{
MONIQUE RADEAU and PAUL BERTELSON \\ Université libre de Bruxelles, B-1050 Bruxelles, Belgium
}

\begin{abstract}
Subjects pointed blindfolded at auditory targets before and after exposure to spatial conflict between the sound of a percussion instrument and images on a TV screen. Four experimental conditions were obtained by combining two levels of realism, in which sound was paired with either the image of the hands playing the instrument or with synchronously modulated light, and two levels of suggestion, in which a dummy loudspeaker from which the subjects had been told the sound would come was placed either in front of the TV screen or on top of the actual hidden loudspeaker. Adaptation occurred in all four conditions, but no difference between them was detected. These results confirm and extend the previous finding that auditory adaptation, unlike the impression of fusion (ventriloquism), is little influenced by cognitive factors.
\end{abstract}

In a recent study (Radeau \& Bertelson, 1977), we undertook to determine the conditions under which pairing of registered locations, the hypothetical mechanism at the basis of cross-modal recalibration, occurs in the case of auditory-visual discordance.

We tried to sort out the effect on auditory adaptation of structural and cognitive factors. For that purpose, the synchronization between auditory and visual data and the degree of realism of the context were manipulated. Ear-hand coordination was measured before and after a period of exposure where the subject monitored an image on a TV screen and sounds coming from a hidden loudspeaker, the two sources being separated in azimuth by $20^{\circ}$. In one experiment, the realistic exposure condition combined the sound of a percussion instrument, bongos, with the image of the hands of the player. The nonrealistic condition was obtained by replacing the sight of the hands by diffuse light modulated by the sound. In another experiment, the exposure featured a voice and either the face of the speaker or light modulated by the voice, in both situations either with exact synchronization between sound and image or with some asynchrony. Desynchronization reduced recalibration significantly. The degree of realism of the situation was found to be irrelevant.

The negative result regarding realism, however, had to be viewed with some caution. First, in some studies, cognitive factors have been shown to influence adaptation to cross-modal discordance. In the case of visuo-proprioceptive conflict, Welch (1972) found that amount of adaptation was strongly dependent on what the subjects were led to believe regarding the identity of the finger they saw in a position different from the felt one-whether it was their own finger or another one. For auditory-visual conflict, Radeau and Bertelson (1974) found that amount of adaptation was influenced, although in a rather complicated way, by descriptions of the situation suggesting either a common origin or independent origins for auditory and visual data.

In the study under discussion, on the other hand, the information regarding the respective origin of sound and image was rather ambiguous. On the one hand, the displaced loudspeaker was not effectively hidden, but simply placed under a rather obvious cover. Sophisticated subjects could thus easily be led to suspect that the sound came from that position. On the other hand, answers to questions asked after completion of testing revealed that many subjects thought that the monitor contained an in-built loudspeaker through which the sound was delivered. The situation thus involved features both favorable and unfavorable to an interpretation in terms of a common origin. Still to be considered was the possibility that contextual realism could become effective in combination with a more systematic suggestion of common origin.

The aim of the present study was to investigate this possibility. The experiment involved four conditions in which semirealistic or nonrealistic situations were presented together with a suggestion of common or of different origin.

\section{METHOD}

The experimental arrangement was the same as in Experiment 1 of Radeau and Bertelson (1977). In brief, the subject was seated in front of a large horizontal panel. His head was held in position by a bite. During test trials, he was blindfolded. On each trial, a $5-\mathrm{sec}$ sequence of bongo sound was delivered through a small movable loudspeaker, $6 \mathrm{~cm}$ in diameter, which the experi- 
menter placed in one of five positions: in the median plane or either $3^{\circ}$ or $6^{\circ}$ to its left or to its right. The subject was asked to point in the direction of the sound by moving his right index finger along the surface of the panel until it reached a fence consisting of a semicircular plastic rod (radius $45 \mathrm{~cm}$ ) lined on the outside by a graduation on which the position reached by the finger could be read. During conflict exposure periods, a TV monitor was placed immediately behind the fence with its axis $20^{\circ}$ to the left or to the right of the median plane. The display conditions were the same as in the previous experiment. In the $\mathrm{B}-\mathrm{H}$ (for bongos-hands) condition, the bongos and the hands of the player were seen on the screen while the corresponding sounds were played in the loudspeaker. In the B-ML (for bongosmodulated light) condition, the illumination of the screen was 'modulated by the sound itself and the screen was covered with tracing paper, so that diffuse light flashing at the rhythm of the sound-about 1 flash/beat-was seen. In both situations, the subject was told that sounds had occasionally been added or deleted and that his task was to detect such occurrences and to report them at the end of each 1-min exposure period.

There were two main departures from the procedure of the previous experiment. Both concern the exposure periods. (1) The sound was delivered through a loudspeaker located in the median plane at the bottom of a $2-\mathrm{cm}$-diam hole in the panel. This loudspeaker was completely invisible, since the whole panel was covered by a homogeneous black cloth. (2) The movable loudspeaker, the same that was used to deliver the sound on test trials, was placed in full view either in front of the TV monitor, in its axis, or in the median position. It never transmitted any sound during conflict exposure periods, but the subjects were told that it was the actual source of the sounds they heard. They were also informed that the monitor contained no loudspeaker. Two different suggestion conditions were thus created: the SO (for same origin) condition with the dummy loudspeaker in front of the screen and the DO (for different origin) condition with the dummy loudspeaker in the same azimuthal direction as the real one (i.e., in the median plane).

The combination of two display conditions (B-H and B-ML) and two suggestion conditions ( $\mathrm{SO}$ and $\mathrm{DO}$ ) provided four experimental conditions. Each of eight subjects did one session under each of these four conditions. The order of conditions was balanced in a Latin square. Four subjects worked with the monitor on the left throughout, and the other four with the monitor on the right.

Each session started with 30 test trials (pretests) and proceeded with two series of eight 1 -min conflict exposure periods, each followed by 15 test trials (posttests). The number of pre- and of posttests was thus double that in the previous experiment. The change was introduced to make the measures more reliable.

Eight volunteer students served as subjects. They were paid 100 Belgian Francs per session for their participation.

\section{RESULTS}

Aftereffects were measured, as usual, by subtracting mean error in posttests from mean error in pretests. Shifts were called positive when they went in the direction of the discordance.

As appears in Table 1, significant aftereffects are observed in each of the four conditions.

Mean individual aftereffects in the four conditions were submitted to a three-way analysis of variance. Neither realism $(F<1)$, nor suggestion $(F<1)$, nor subjects $[F(7,7)=1.17]$ were significant sources of variation. The Realism by Suggestion interaction was also nonsignificant. It can be observed that the corresponding contrast has the opposite value from the one hypothesized: the effect of realism is larger in the DO condition than in the SO condition, where it is, in fact, negative.

\section{DISCUSSION}

No effect of cognitive variables on auditory adaptation to auditory-visual discordance has been detected in the present experiment: neither suggestion of a common origin nor contextual realism affected amount of adaptation, which fluctuated about the $3^{\circ}$ value that we have typically found in our previous studies, after exposure to spatial discordance between synchronized auditory and visual data. In particular, the notion that realism could become an effective factor, provided it was combined with adequate suggestion, has received no support whatsoever.

We have, thus, now rather convincing evidence that adaptation to auditory-visual discordance is little affected by cognitive variables. On the other hand, it is strongly dependent on the structural condition of common fate, as the previous study (Radeau \& Bertelson, 1977) showed.

In the same study, data were obtained which confirmed the suggestion in the literature that ventriloquism, i.e., the impression of fusion of discordant visual and auditory data, is influenced by both cognitive and structural factors. The conclusion, which was then considered tentatively, that consciously perceived fusion and the preconscious pairing operation at the basis of recalibration might be different processes obeying different principles, can now be stated much more firmly.

The data mentioned in the introduction, which suggested an influence of cognitive factors on adaptation to cross-modal conflict, are not necessarily in contradiction with the present ones, since they were obtained with different modalities. Welch (1972) worked with visuo-proprioceptive discordance, and in Radeau and Bertelson's (1974) study, the difference between the two opposite suggestion conditions went in the direction of the suggestion for visual

Table 1

Overall Results

\begin{tabular}{lcccc}
\hline \multicolumn{2}{c}{ Condition } & Mean After- & p (one- \\
Display & Suggestion & effect* & SD* & tailed t test) \\
\hline B-H & SO & 2.44 & 2.82 & .05 \\
B-ML & SO & 3.59 & 2.12 & .005 \\
B-H & DO & 2.92 & 1.66 & .005 \\
B-ML & DO & 2.32 & 2.26 & .025 \\
\hline
\end{tabular}

Note-B-H=bongos-hands; $\quad B-M L=$ bongos-modulated light. $S O=$ suggestion of same origin (dummy loudspeaker in front of $T V$ screen); DO = suggestion of different origin (dummy loudspeaker in direction of actual sound).

*In degrees. 
pointing only. ${ }^{1}$ It is thus possible that the present results would not apply to all other intermodal coordinations.

\section{REFERENCES}

Radeau, M., \& Bertelson, P. The after-effects of ventriloquism. Quarterly Journal of Experimental Psychology, 1974, 26, 63-71.

Radeau, M., \& Bertelson, P. Adaptation to auditory-visual discordance and ventriloquism in semirealistic situations. Perseption \& Psychophysics, 1977, 22, 137-146.
Welch, R. B. The effect of experienced limb identity upon adaptation to simulated displacement of the visual field. Perception \& Psychophysics, 1972, 12, 453-456.

\section{NOTE}

1. For auditory pointing, the difference went in the other direction, while the control no-suggestion condition inexplicably produced less adaptation than either of the opposite suggestion conditions.

(Received for publication October 3, 1977; accepted February 23, 1978.) 